\title{
Metastatic Pancreatic Carcinoma and Experience with FOLFIRINOX - a Cross Sectional Analysis From a Developing Country
}

\author{
Muhammad Nauman Zahir ${ }^{1 *}$, Adnan Abdul Jabbar ${ }^{2}$
}

\begin{abstract}
Background: Pancreatic cancer is the fourth leading cause of cancer related death with median survival ranging from 3 to 6 months for metastatic disease. Palliative chemotherapy has been the backbone of treatment in advanced stage and has evolved over time. Data pertaining to the disease are scarce from our part of the world where treatment poses a significant challenge due to lack of resources. Materials and Methods: A retrospective chart review was performed for all patients presenting with stage IV pancreatic carcinoma at a tertiary care hospital in Karachi, Pakistan between January 2008 and December 2012. Data were collected using a pre-designed, coded questionnaire looking at patient characteristics, treatment given and outcome. Results: 101 patients were found to be eligible. Mean age was 56.7 \pm 12.8 years, the male to female ratio was 2:1 and most patients had a good performance status. More than half of the tumors were located in the head $(57 \%, n=58)$ and almost all were adenocarcinomas $(95 \%, n=96)$. Some $58 \%(n=59)$ received first line chemotherapy of which $49 \%(n=29)$ received gemcitabine-based regimens and 39\% $(\mathrm{n}=\mathbf{2 3})$ received FOLFIRINOX. The median progression free survival for gemcitabine based treatment was 2.9 months (IQR=1.6-5.6) as opposed to 7.3 months (IQR=4.5-9.2) for FOLFIRINOX $(\mathrm{P}=0.02)$. Median overall survival was 4.9 months $(\mathrm{IQR}=2.3-9.5)$ for first line gemcitabine based treatment and 10.5 months $(I Q R=7.0-13.2)$ for first line FOLFIRINOX therapy $(P=0.002)$. Patients on FOLFIRINOX had better survival across all subgroups. Inpatient admissions and dose reductions were more frequent with FOLFIRINOX but the difference between the two regimens was not statistically significant. FOLFIRINOX could be successfully administered as outpatient therapy to a number of patients. Conclusions: FOLFIRINOX remains a suitable first line option in patients with metastatic pancreatic cancer with good performance status even in a resource-poor country where diagnostic and supportive care facilities may be less than optimal and cost is a limitation.
\end{abstract}

Keywords: Pancreatic - cancer - gemcitabine, FOLFIRINOX

Asian Pac J Cancer Prev, 16 (14), 6001-6006

\section{Introduction}

Pancreatic carcinoma is the fourth leading cause of cancer related death in the Western world and the third most common malignancy of the gastrointestinal tract (Siegel, Naishadham and Jemal, 2013). The aggressive nature of the disease coupled with advanced stage at diagnosis in most cases, results in 5 year survival rate of less than 5\% (Jemal et al., 2005). Surgical resection offers the only chance of cure but less than 15 to $20 \%$ are resectable at initial diagnosis (Parvez and Dawood, 2003). Median survival ranges from 8 to 12 months for locally advanced unresectable disease and 3 to 6 months for patients presenting with metastasis (Siegel et al., 2013).

Chemotherapy has traditionally been the backbone of treatment for advanced pancreatic carcinoma and has evolved over time with gemcitabine based treatment occupying the niche of "standard of care" until recently
(Di Marco et al., 2010). Gemcitabine therapy was established as standard of care in advanced pancreatic carcinoma back in 1997 (Burris et al., 1997) and since then numerous studies have validated a median overall survival of 6 months in advanced disease when the drug has been used as a single agent (Conroy and Mitry, 2011). Doublet chemotherapy has some benefit in response rate (RR) and progression free survival (PFS) without any clear difference in overall survival (OS) (Cunningham et al., 2009). One promising emerging chemotherapeutic option is the nab-Paclitaxel plus Gemcitabine combination which has been shown to be effective (Von Hoff et al., 2013).

Improvement in the understanding of the molecular biology of pancreatic cancer has led to an increased effort to develop new management strategies. It is hoped that these modalities will help improve the outcome of advanced pancreatic carcinoma in the future (Valsecchi et al., 2014). 
In May 2011, Conroy et al. published their experience with FOLFIRINOX (combination chemotherapy comprising oxaliplatin, irinotecan, folinic acid and 5-fluorouracil) where they reported a significant survival benefit in comparison to Gemcitabine based chemotherapy (Conroy et al., 2011).Although it has now been established in the West as the preferred regimen for patients with metastatic pancreatic cancer with good performance status and a normal serum bilirubin level (Conroy et al., 2011), no report from Asian trials or documented anecdotal experience of its use from South Asia is available (Conroy et al., 2013). A recently reported study has evaluated XELOX as $2^{\text {nd }}$ line therapy in an Asian population with encouraging anti-tumor activity and manageable toxicity, laying the foundation for further research exploring FOLFIRINOX as a potential first line regimen in this population (Bayoglui et al., 2014).

Pakistan with the sixth highest world population and increasing burden of cancer including pancreatic carcinoma has a similar trend, with most patients presenting at an advanced stage. There is a paradigm shift towards FOLFIRINOX as the preferred treatment regimen in appropriately selected patients but no report of the outcome, adverse events and challenges with the treatment are available. Maintaining intravenous lines for 5-FU infusion pump with high incidence of infection due to poor hygiene, increased cost of FOLFIRINOX over gemcitabine and poor educational background were reasons for reservation of this regimen in our population. Generally, there is dearth of data regarding experience with FOLFIRINOX from South Asia where the documentation of this experience is also of paramount importance because of the genetic and cultural variability of the region from the West.

This study was undertaken to estimate the burden of stage IV pancreatic carcinoma at a major tertiary care center in Karachi, Pakistan. We aimed to reflect upon the management options used for metastatic disease at our center, specifically highlighting differences in responses between major treatment groups and the survival patterns of these patients despite the local challenges.

\section{Materials and Methods}

\section{Patients}

Retrospective data was collected for adult patients ( $\geq 16$ years) with recently diagnosed (histopathologically or cytologically confirmed) primary metastatic pancreatic carcinoma presenting to the Aga Khan University Hospital (AKUH), Karachi between January 1, 2007 and December 31, 2012. Patients were identified using the International Classification of Diseases (ICD) coding system and the cancer registry at AKUH. Patients had to be treatment naive with no prior history of malignancy to be included in the study.

Data was collected using a pre-designed and coded questionnaire approved by the institutional Ethical Review Committee. Details of demographics, symptomatology, risk factors, diagnostic modalities used, histopathological features, and outcome were collected for all patients.

Follow up records, data pertaining to therapies and dates of last contact were also recorded.

\section{Statistical analysis}

Statistical Package for Social Sciences (SPSS) version 19 was used to perform data analysis on 101 eligible patients. Data was reported as mean \pm standard deviation for continuous variables and proportions and percentages for categorical data. Continuous variables not following the normal distribution were reported as medians along with inter-quartile ranges.

Analysis was performed on the entire cohort followed by a subgroup analysis according to the 2 major chemotherapy regimens employed (Gemcitabine based regimens $v s$. FOLFIRINOX). Categorical variables in the subgroups were compared using the Pearson Chi Square test or Fischer's test while the Student's t-test was used to compare continuous data. Median PFS and median OS were calculated in the two groups and Kaplan Meier curves were generated. Median PFS in the two groups was compared using the log-rank test while median OS was compared using the Mann-Whitney test. P value less than 0.05 was considered significant.

\section{Results}

\section{Patients}

101 eligible patients with metastatic pancreatic carcinoma presented to our institution between January 1, 2007 and December 31, 2012 [Table 1]. The mean age of the study population was $56.7 \pm 12.8$ years and the male to female ratio was $2: 1$. Most patients had a good performance status at diagnosis $(84 \%, \mathrm{n}=85)$. More than half of the tumors were located in the head of the pancreas $(57 \%, \mathrm{n}=58)$ and almost all were adenocarcinomas $(95 \%$, $\mathrm{n}=96)$. The most common clinical presentations included abdominal pain $(95 \%, n=96)$, weight loss $(88 \%, n=89)$, icterus $(55 \%, \mathrm{n}=55)$ and worsening of established type 2 Diabetes $(40 \%, \mathrm{n}=40)$. Deep venous thrombosis was a presenting feature in $23 \%$ of patients $(n=23)$.

On subgroup analysis, the baseline characteristics, clinical presentations and laboratory parameters were similar between the two groups divided according to the two major chemotherapy regimens employed (Table 1). The only notable difference was the presence of a larger number of patients with an abnormal serum level of CA-19-9 in the Gemcitabine group as compared to the FOLFIRINOX group ( $72 \%$ vs. $48 \%$ ). The relative absence of patients with jaundice in the FOLFIRINOX group can be attributed to the pharmacological limitation of using hepatotoxic agents in patients with grossly abnormal bilirubin levels (11).

\section{Treatment}

Almost half of the study population $(42 \%, n=42)$ presented at a stage where chemotherapeutic intervention was not possible and received palliative surgical intervention $(n=10)$ or comfort care $(n=32)$. Of the remaining 59 patients who were clinically suitable to receive first line chemotherapy, $49 \%(n=29)$ received Gemcitabine based regimens, 39\% $(n=23)$ received FOLFIRINOX whereas $12 \%(n=7)$ were treated with other 
Metastatic Pancreatic Carcinoma and Experience with FOLFIRINOX - a Cross Sectional Analysis from a Developing Country chemotherapeutic regimens.

The median number of cycles received by patients in the Gemcitabine group were 4 (IQR=3-7.5) as opposed to $16(\mathrm{IQR}=9-19)$ in the FOLFIRINOX group $(\mathrm{p}<0.001)$. This corresponded to a median duration of 12.8 weeks (IQR=7.4-25.1) for patients on Gemcitabine versus 35.8 weeks (IQR=21.6-41.6) for patients in the FOLFIRINOX group $(\mathrm{p}<0.001)$.

The most common grade 3 and 4 side effects with first line Gemcitabine were elevated Alanine transaminase (ALT) $14 \%(n=4)$, febrile neutropenia $10 \%(n=3)$ and thrombocytopenia $7 \%(n=2)$ whereas for first line FOLFIRINOX, these were febrile neutropenia $(22 \%, n=5)$, thrombocytopenia $(22 \%, \mathrm{n}=5)$, peripheral neuropathy $(9 \%, n=2)$ and diarrhea $(9 \%, n=2)$ [Table 2].

Although the frequency of side effects, dose reductions and patients requiring inpatient admissions were more common in the group receiving FOLFIRINOX, the difference between the 2 groups was not found to be statistically significant [Table 2].

Only 18 patients $(18 \%)$ received any second-line chemotherapy out of which 7 (39\%) received Gemcitabine based therapy, 5 (28\%) received Capecitabine based treatment and $4(22 \%)$ were given FOLFIRINOX. The number of patients receiving second line chemotherapy was too small for meaningful analysis.

\section{Outcome}

At 6 months followup, only $24 \%$ of patients $(n=24)$ from the entire cohort were alive. All 42 patients who had not received any chemotherapy had died. At the one year interval the percentage of surviving patients had dropped to an even more dismal $13 \%(n=13)$.

On subgroup analysis according to the two major chemotherapy groups, only $24 \%(n=7)$ patients in Gemcitabine group were alive as opposed to $61 \%(n=14)$ on FOLFIRINOX at the 6 month interval $(\mathrm{p}<0.001)$. This difference in overall survival remained statistically significant at 1 year followup as well (17\% Gemcitabine vs. $26 \%$ FOLFIRINOX, $\mathrm{p}=0.008)$.

Table 1. Baseline Characteristics of the Study Population

\begin{tabular}{|c|c|c|c|}
\hline Variables & $\begin{array}{c}\text { All metastatic } \\
\text { pancreatic cancers } n=101\end{array}$ & $\begin{array}{l}1^{\text {st }} \text { line Gemcitabine } \\
\text { based treatment } n=29\end{array}$ & $\begin{array}{l}1^{\text {st }} \text { line FOLFIRINOX } \\
n=23\end{array}$ \\
\hline Age, Mean (SD) & $56.7(12.8)$ & $54.4(12.4)$ & $51.9(10.5)$ \\
\hline$\leq 40(\%)$ & $13(12.9)$ & $4(13.8)$ & $4(17.4)$ \\
\hline$>40(\%)$ & $88(87.1)$ & $25(86.2)$ & $19(82.6)$ \\
\hline Gender, Male (\%) & $69(68.3)$ & $19(65.5)$ & $14(60.9)$ \\
\hline \multicolumn{4}{|l|}{ ECOG Performance Status } \\
\hline 0 to 2 & $85(84.2)$ & $28(96.6)$ & $23(100)$ \\
\hline 3 to 4 & $16(15.8)$ & $1(3.4)$ & - \\
\hline \multicolumn{4}{|l|}{ Site of Tumor } \\
\hline Head (\%) & $58(57.4)$ & $14(48.3)$ & $13(56.5)$ \\
\hline Body/tail (\%) & $43(42.6)$ & $15(51.7)$ & $7(43.5)$ \\
\hline \multicolumn{4}{|l|}{ Diagnosis } \\
\hline Adenocarcinoma (\%) & $96(95)$ & $29(100)$ & $22(95.7)$ \\
\hline Other $(\%)$ & $5(5)$ & - & $1(4.3)$ \\
\hline \multicolumn{4}{|l|}{ Symptoms } \\
\hline Abdominal Pain (\%) & $96(95)$ & $27(93.1)$ & $22(95.7)$ \\
\hline Weight Loss $(\%)$ & $89(88.1)$ & $26(89.7)$ & $17(73.9)$ \\
\hline Jaundice $(\%)$ & $55(54.5)$ & $14(48.3)$ & $10(43.5)$ \\
\hline Worsening of Diabetes (\%) & $40(39.6)$ & $9(31)$ & $9(39.1)$ \\
\hline $\operatorname{DVT}(\%)$ & $23(22.8)$ & $6(20.7)$ & $3(13.0)$ \\
\hline \multicolumn{4}{|l|}{ Laboratory Parameters } \\
\hline Total bilirubin $>2(\%)$ & $48(47.5)$ & $12(41.4)$ & $4(17.4)$ \\
\hline Normal CA-19-9 & $27(26.7)$ & $8(27.6)$ & $12(52.2)$ \\
\hline
\end{tabular}

Table 2. Grade 3-4 Adverse Effects, Dose Reduction and In-Patient Admission Data

\begin{tabular}{lccc}
\hline Variables & $\begin{array}{c}\text { All metastatic pancreatic } \\
\text { cancers receiving } 1^{\text {st }} \text { line } \\
\text { chemotherapy }(\mathrm{n}=52)\end{array}$ & $\begin{array}{c}1^{\text {st }} \text { line Gemcitabine } \\
\text { based treatment } \\
(\mathrm{n}=29)\end{array}$ & $\begin{array}{c}1^{\text {st }} \text { line } \\
\text { FOLFIRINOX } \\
(\mathrm{n}=23)\end{array}$ \\
\hline Major Side Effects of Chemotherapy & & & $\mathrm{p}$ value \\
$\quad$ Thrombocytopenia (\%) & $7(13.5)$ & $2(6.9)$ & $5(21.7)$ \\
Febrile Neutropenia (\%) & $8(15.4)$ & $3(10.3)$ & $6(21.7)$ \\
Peripheral Neuropathy (\%) & $2(3.8)$ & $0(0)$ & $7(21.7)$ \\
Diarrhea (\%) & $3(5.8)$ & $1(3.4)$ & $8(21.7)$ \\
Mucositis (\%) & $1(1.9)$ & $0(0)$ & $9(21.7)$ \\
AKI (\%) & $2(3.8)$ & $4(13.8)$ & $10(21.7)$ \\
Elevated ALT (\%) & $5(9.6)$ & $2(6.9)$ & $11(21.7)$ \\
Dose Reduction Needed (\%) & $7(13.4)$ & $8(27.6)$ & $5(21.7)$ \\
Admission Required (\%) & $15(28.8)$ & & $7(30.4)$ \\
\hline
\end{tabular}




\begin{tabular}{llccr}
\hline Variables & & $\begin{array}{c}\text { Median survival } \\
\text { All cases (n=101) }\end{array}$ & $\begin{array}{c}\text { Median Survival } \\
\text { Gemcitabine (n=29) }\end{array}$ & $\begin{array}{c}\text { Median Survival } \\
\text { FOLFIRINOX(n=23) }\end{array}$ \\
\cline { 3 - 5 } Age & Months (IQR) & Months (IQR) & Months (IQR) \\
\hline \multirow{2}{*}{ Gender } & Entire cohort & $4.7(2.1-8.6)$ & $4.9(2.3-9.5)$ & $10.5(7.0-13.2)$ \\
& $\leq 40$ & $6.9(3.0-11.2)$ & $7.9(3.0-15.4)$ & $7.1(2.8-10.2)$ \\
ECOG & $>40$ & $4.6(1.9-8.3)$ & $4.9(2.3-7.9)$ & $10.8(7.2-15.1)$ \\
& Male & $4.1(1.8-8.0)$ & $5.1(4.3-11.3)$ & $10.6(6.3-13.7)$ \\
Total Bilirubin & Female & $4.1(1.8-8.0)$ & $2.2(1.2-5.1)$ & $10.6(6.3-13.7)$ \\
& ECOG 0-2 & $5.3(2.4-10.4)$ & $4.9(2.4-10.0)$ & $10.5(7.0-13.2)$ \\
CA-19-9 & ECOG 3-4 & $1.8(1.2-2.5)$ & $2.3(\mathrm{n}=1)$ & - \\
& $<2 \mathrm{mg} / \mathrm{dl}$ & $5.4(2.2-10.1)$ & $4.2(2.2-5.0)$ & $10.4(7.0-11.9)$ \\
Site of tumor & $\geq 2 \mathrm{mg} / \mathrm{dl}$ & $3.2(1.8-7.3)$ & $9.0(4.8-15.5)$ & $13.0(6.5-16.6)$ \\
& Normal & $10.0(2.5-15.3)$ & $4.9(2.3-7.9)$ & $8.8(6.2-11.1)$ \\
& Abnormal & $3.8(1.8-6.4)$ & $5.7(2.5-15.5)$ & $13.0(6.5-16.6)$ \\
& Head & $5.1(2.3-10.1)$ & $2.5(1.4-6.2)$ & $10.8(6.2-15.2)$ \\
& Body/tail & $3.2(1.7-8.4)$ & & $10.3(7.2-11.6)$ \\
\hline
\end{tabular}

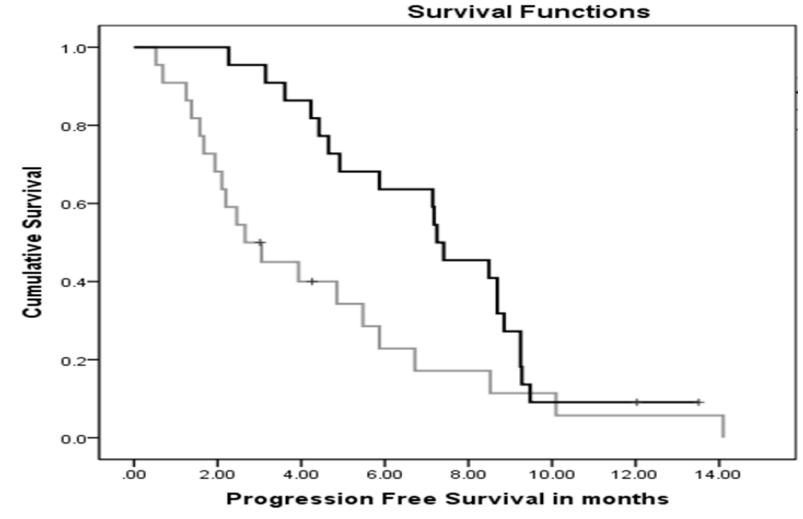

Figure 1. Kaplan-Meier Curve for Progression Free Survival (PFS)

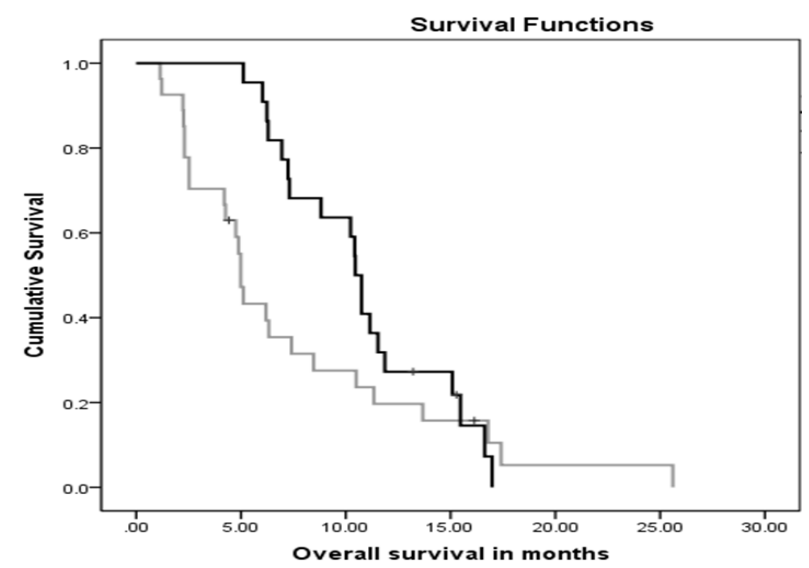

Figure 2. Kaplan-Meier Curve for Overall Survival (OS)

The median PFS for patients on first line Gemcitabine based chemotherapy was 2.9 months (IQR=1.6-5.6) as opposed to 7.3 months (IQR=4.5-9.2) for patients on first line FOLFIRINOX ( $\mathrm{p}=0.003)$ [Figure 1].

The median OS was 4.9 (IQR=2.3-9.5) months for the Gemcitabine and 10.5 (IQR=7.0-13.2) months for the FOLFIRINOX group ( $\mathrm{p}=0.002)$ [Figure 2]. The median OS for the entire cohort was 4.7 months (IQR=2.1-8.6).

Patients on FOLFIRINOX had better survival across all subgroups when compared with patients who had received Gemcitabine [Table 3].

\section{Stratified subgroup analysis}

A stratified subgroup analysis was carried out to look for the effect of age, gender, ECOG performance status, total bilirubin, CA 19-9 and site of tumor on outcome within each therapeutic sub-group. No statistically significant contribution of these variables was noted on outcome during analysis [Table 3]. It is possible that a difference may not have been elucidated due to the small number of patients in the sub-groups.

\section{Discussion}

Although small, our study is the first describing experience with FOLFIRINOX in the South Asian population. The results from our study reiterate a significant survival benefit with FOLFIRINOX when compared to gemcitabine based treatment. The approximate doubling of both PFS (7.3 vs. 2.9 months) and OS (10.5 vs. 4.9 months) with FOLFIRINOX vs. Gemcitabine based therapy mirrors the results of the pivotal paper by Conroy et al. (2011) and is similar to the OS reported in other Western studies (Peddi et al., 2012; Gunturu et al., 2013). The survival at one year in the FOLFIRINOX group in our study was lower than the one reported in Conroy's study (48\% vs. 26\%) which may be secondary to the lower number of patients in our study.

These results suggest that the therapeutic benefits of FOLFIRINOX over Gemcitabine hold true for the Asian population as well as in our clinical setting. This coupled with the observation that FOLFIRINOX confers approximately the same benefit across all patient subgroups further adds to the value of the regimen [Table 3]. The only subgroup of patients which showed an inferior survival amongst patients receiving FOLFIRINOX in our study was that of $<40$ year olds. However no definitive inference can be made due to the small number of patients in this subgroup.

One may argue that a higher proportion of patients in the FOLFIRINOX group in our study had a normal CA 19-9 at presentation and may have been responsible for the better prognosis seen with this chemotherapy regimen as it has been reported to be a predictor of better survival 
Metastatic Pancreatic Carcinoma and Experience with FOLFIRINOX - a Cross Sectional Analysis from a Developing Country

in chemo-naive patients (Berger et al., 2004; Waraya et al., 2009; Ballehaninna and Chamberlain, 2012). This hypothesis is however refuted by the fact that the survival benefit inferred by the normal serum CA 19-9 levels in chemo-naive metastatic pancreatic carcinoma patients, was lost when patients received any chemotherapy [Table 3]. This is an interesting observation though and one which can be further explored.

Another interesting observation in the group which received first line Gemcitabine was patients with a raised bilirubin level and those with tumors of the head of the pancreas fared better than those with normal bilirubin and tumors elsewhere in the pancreas. One may speculate that patients with tumors of the head of the pancreas would more likely present early secondary to extrinsic biliary obstruction and jaundice, resulting in better outcome, but in the end these were all stage IV patients.

One of the major limitations of FOLFIRINOX has been the higher frequency of adverse effects observed with the regimen (Ko 2011). The skepticism associated with FOLFIRINOX use has somewhat been dispelled with the use of the regimen in several studies in the West which despite indicating a clearly significant increase in adverse events with its use, also prove that these are easily manageable with appropriate therapy (Conroy et al., 2011; Peddi et al., 2012; Gunturu et al., 2013). A similar trend was seen in our study where although the frequency of grade 3 and 4 side effects were higher in the FOLFIRINOX group, they were not significantly different from the Gemcitabine group and were manageable with adequate treatment when they did occur. Also patients received more weeks of treatment in FOLFININOX group compared to Gemcitabine based chemotherapy. This highlights the fact that the regimen is reasonably well tolerated in our population and in light of the statistically significant survival benefit conferred; the slightly unfavorable toxicity profile may well be acceptable to patients and clinicians worldwide.

Experimentation with modifications to the FOLFIRINOX regimen have been carried out to reduce toxicity without compromising on the efficacy and a recent study by Mahaseth et al. which deleted 5-FU bolus from the regimen while employing routine use of GCSF, has shown to be better tolerated while achieving disease control in most patients (Mahaseth et al., 2013). Researchers elsewhere have employed reduced median relative doses of FOLFIRINOX but have managed to maintain efficacy (Gunturu et al., 2013). These modifications may eventually result in further improving the toxicity profile of the regimen making it more palatable.

An important factor dictating the acceptance of FOLFIRINOX in a resource poor country like Pakistan is the cost associated with its use. Although the regimen has been proclaimed as cost-effective (Mahaseth et al., 2013), the substantial out of pocket disbursement necessary in our country has the potential of limiting its use to the affluent only. An estimate of the difference in cost calculated at the end of six weeks of therapy between the two regimens indicates FOLFIRINOX to be twice as expensive as Gemcitabine based treatment (\$1524 vs. \$762). Until recently FOLFIRINOX was given solely as inpatient treatment, but with the recent availability of outpatient infusion pumps we have transitioned the regimen to an out-patient setting without a distinct increase in complication rates or side effects in an economically transitioning country with less than optimal hygienic conditions. This change has further reduced the cost of regimen and it is possible that modifications in the regimen itself (e.g. deletion of 5-FU bolus) may make it even more affordable in the near future.

Our study has certain limitations. One of the most imminent limitations is the retrospective nature of collection of data through hospital records. This has the potential for incomplete data collection due to missing information in the charts. In our defence, most of our variables are laboratory investigations, treatment related variables and survival data which are documented in detail at our institution and hence are less likely to create bias because of incomplete information. Secondly, this is a single center study and it can be argued that the results cannot be generalized to the local population at large. The fact that metastatic pancreatic carcinoma is a rare disease and Aga Khan University is the major tertiary care center catering to patients from all over the country, we can speculate to generalize the result as the numbers in each individual subgroup seems reasonable. Finally, an element of bias that could not be controlled was the difference in calendar time for receiving the two major chemotherapy regimens. Gemcitabine based chemotherapy was solely prescribed till 2 years ago when it was the standard of care whereas FOLFIRINOX became practice recently. This difference has the potential of skewing the survival results in favor of FOLFIRINOX as part of the survival benefit may be due to improved supportive care and other secular trends over time.

In conclusion, our study attempts to fill the void of information currently present pertaining to pancreatic cancer in our country. It highlights the baseline characteristics and outcomes of patients presenting with metastatic disease to our institution. To the best of our knowledge this is the first study from South Asia narrating experience with FOLFIRINOX and hence it is also the first to compare the regimen with Gemcitabine based chemotherapy.

We strongly believe that the results of this study should be able to instill enough confidence in physicians in the region to use the regimen as first line treatment in appropriately selected patients even in a resource challenged country such as ours.

\section{Acknowledgements}

The authors would like to thank Ms. Saba Malik and Ms. Saadia Pervaiz from the Cancer registry of Aga Khan University Hospital for their help in identifying patients. We would also like to acknowledge the help provided by Dr. Quratulain Shaikh in the statistical analysis of this manuscript.

\section{References}

Ballehaninna UK, Chamberlain RS (2012). The clinical utility of 
serum CA 19-9 in the diagnosis, prognosis and management of pancreatic adenocarcinoma: An evidence based appraisal. J Gastrointest Oncol, 3, 105-19.

Bayoglui IV, Varoli U, Yildizi I, et al (2014). Second-line capecitabine and oxaliplatin combination for gemcitabineresistant advanced pancreatic cancer. Asian Pac J Cancer Prev, 15, 7119-23.

Berger AC, Meszoely IM, Ross EA, Watson JC, Hoffman JP (2004). Undetectable preoperative levels of serum CA 19-9 correlate with improved survival for patients with resectable pancreatic adenocarcinoma. Ann Surg Oncol, 11, 644-9.

Burris HA 3rd, Moore M J,Andersen J, et al (1997). Improvements in survival and clinical benefit with gemcitabine as first-line therapy for patients with advanced pancreas cancer: a randomized trial. J Clin Oncol, 15, 2403-13.

Conroy T, Desseigne F, Ychou M, et al (2011). FOLFIRINOX versus gemcitabine for metastatic pancreatic cancer. $N$ Engl J Med, 364, 1817-25.

Conroy T, Mitry E (2011). Chemotherapy of metastatic pancreatic adenocarcinoma: challenges and encouraging results. Bull Cancer, 98, 1439-46.

Conroy T, Gavoille C, Samalin E, Ychou M, Ducreux M (2013). The role of the FOLFIRINOX regimen for advanced pancreatic cancer. Curr Oncol Rep, 15, 182-9.

Cunningham D, Chau I, Stocken DD, et al (2009). Phase III randomized comparison of gemcitabine versus gemcitabine plus capecitabine in patients with advanced pancreatic cancer. J Clin Oncol, 27, 5513-8.

Di Marco M, Di Cicilia R, Macchini M, et al (2010). Metastatic pancreatic cancer: Is gemcitabine still the best standard treatment? (Review). Oncology reports, 23, 1183-92.

Gunturu KS, Yao X, Cong X, et al (2013). FOLFIRINOX for locally advanced and metastatic pancreatic cancer: single institution retrospective review of efficacy and toxicity. Med Oncol, 30, 361.

Jemal A, Murray T, Ward E, et al (2005). Cancer statistics, 2005. CA Cancer J Clin, 55, 10-30.

Ko, A. H. (2011) FOLFIRINOX: a small step or a great leap forward? J Clin Oncol, 29, 3727-9.

Mahaseth H, Brutcher E, Kauh J, et al (2013). Modified FOLFIRINOX regimen with improved safety and maintained efficacy in pancreatic adenocarcinoma. Pancreas, 42, 13115.

Parvez T, Dawood T (2003). Pancreatic cancer: new strategies available, but long battle ahead. J Coll Physicians Surg Pak, 13, 303-4.

Peddi PF, Lubner S, McWilliams R, et al (2012). Multiinstitutional experience with FOLFIRINOX in pancreatic adenocarcinoma. JOP, 13, 497-501.

Siegel R, Naishadham D, Jemal A (2013). Cancer statistics, 2013. CA Cancer J Clin, 63, 11-30.

Valsecchi ME, Diaz-Canton E, de la Vega M, Littman SJ (2014). Recent Treatment Advances and Novel Therapies in Pancreas Cancer: A Review. J Gastrointest Cancer.

Von Hoff DD, Ervin T, Arena FP et al (2013). Increased survival in pancreatic cancer with nab-paclitaxel plus gemcitabine. N Engl J Med, 369, 1691-703.

Waraya M, Yamashita K, Katagiri H, et al (2009). Preoperative serum CA19-9 and dissected peripancreatic tissue margin as determiners of long-term survival in pancreatic cancer. Ann Surg Oncol, 16, 1231-40. 